\title{
BIBLIOGRAPHIE DE BERNARD BARBIER
}

1. Petite histoire de la Durance dans le Gapençais, B.S.E.H.A., 1953.

2. Géographie de l'industrie atomique, I.G., 1956, 5.

3. Le problème de l'eau en Provence, B.S.G.M., 1960.

4. Irrigation et développement agricole en Moyenne et Basse-Durance alpestre, Méd., 1962, 2.

5. L'évolution démographique en Provence-Côte-d'Azur, B.S.G.M., 1962.

6. Les centres élémentaires à fonction urbaine des Alpes du Sud, Méd., 1964, 4.

7. Méthodes d'étude des résidences secondaires, l'exemple des Basses Alpes, Méd., 1965, 2.

8. Puget Théniers et son rôle régional, B.S.G.S.S., 1966.

9. Tourisme et emploi, Méd., 1966, 2.

10. Les zones d'attraction commerciale de la Région Provence-Côte-d'Azur, en collaboration avec N.Delefortrie-Soubeyroux et A Strazzieri, no spécial de la R.C.C.M., 1966, un vol. de 204 p. et 100 cartes; édité en 1967 chez Gauthier-Villars, Paris; atlas publié à part.

11. Logements de vacances et résidences secondaires dans la Région Provence-Côte d'Azur, B.A.G.F., 1956 et Cahiers du tourisme (C.H.E.T.), 1967, série A, 5.

12. La Foux d'Allos, Cahiers du Tourisme, C.H.E.T., 1967.

13. Vars, une grande station de ski des Alpes du Sud, R.G.A., 1968, 2.

14. Marseille et les Alpes du Sud, R.C.C.M., 1969, 813.

15. La fréquentation des stations de ski sudalpines, Cahiers du Tourisme (C.H.E.T.), 1969.

16. Villes et centres des Alpes du Sud, thèse de doctorat-ès-Lettres et Sciences humaines, Université d'Aix-en-Provence, 1969; éd. Ophrys, Gap, un vol., 422 p.

17. La ville sudalpine, [in:] Actes du Congrès National des Géographes Italiens, 1972.

18. Wetzlar, une ville moyenne allemande, Et.V., no spécial, 1972.

19. La survie des petites villes de montagne, R.G.A., 1972, 2.

20. Essai de mesure du dynamisme des petites villes, en coll., avec G.Veyret, in Recherches Géographiques, Congrès de l'Union Géographique Internationale, Montréal, 1972.

21. Le rôle des petites villes en milieu montagnard, B.A.G.F., mai-juin 1972.

22. Le tourisme dans le Bassin Méditerranéen, in Actes du Colloque franco-italien de Taormine, 1973.

23. Les Alpes, [in:] «Découvrir la France», éd. Larousse, 1973, un vol., en coll.

24. La région touristique, [in:] Actes du Colloque franco-hongrois, Budapest, 1973.

25. La Provence, [in:] «Découvrir la France», éd. Larousse, 1974, un vol., en coll.

26. Pour une géographie du tourisme, [in:] publ. de l’Université de Łódź, 1975.

27. Les transformations de Vars, Méd., 1976, 3.

28. La politique française de la montagne, [in:] Actes du Colloque de Dombaï, 1976, (U.G.I.).

29. La consommation d'espace liée à la croissance urbaine en Provence-Côte d'Azur, Congrès de l'Union Géographique Internationale, Moscou, 1976 (Actes du congrès).

30. La consommation d'espace liée à la croissance urbaine en France, colloque franco-japonais de Tokyo, 1976, publié dans les actes du colloque (Maison franco-japonaise, 1978).

31. Les villes moyennes en France, actes du colloque de géographie urbaine, Łódź, 1977 (en polonais).

32. Le tourisme dans les régions karstiques françaises, Proceedings of the $6^{\text {th }}$ International congress of speleogy, Akademia, Praha, 1977.

33. Le ski dans le monde, Congrès international du Groupe de travail tourisme de l'Union Géographique Internationale, Birmingham, 1977, publié in W.G.S., 1979.

34. Le tourisme en Camargue, R.G.G.R., 1978. 
35. Le rôle de l'industrie dans les villes françaises, actes du colloque de géographie urbaine, Łódź, 1978, (en polonais).

36. Les résidences secondaires et leur rôle dans l'espace rural français, Nor., no spécial (Mélanges Fénelon), 1978.

37. Le tourisme polonais vu par un géographe français, Cahiers scientifiques de l'Université Jagellonne, série géographique, Kraków, 1978.

38. Industrie et développement urbain dans les ports français, actes du colloque de géographie urbaine de Łódź, 1979 (en polonais).

39. Urbanisation et consommation d'espace en France, H.T. du N., 1979, 2; no spécial sur la consommation d'espace liée à la croissance urbaine consacré aux travaux de la commission de géographie urbaine du Comité National Français de Géographie sur ce sujet (Président: B. Barbier).

40. Aspects de la consommation d'espace par les villes en Provence, d'après le système Sirocco, H.T. du N., 1979, 2.

41. Le rôle des villes dans les Alpes du sud, Doc. Fr., 1980.

42. Le thème de la banlieue dans la géographie française, actes du colloque de géographie urbaine de Łódź, 1980, (en polonais).

43. La géographie du ski dans les pays socialistes, R.G.A., 1980, (Mélanges P. Veyret).

44. Tourisme et environnement physico-spatial. Rapport de base présenté au Congrès de l'Association internationale des Experts Scientifiques du Tourisme (A.I.E.S.T.), Palma de Majorque, 1980, éd. Gurten, Berne.

45. La géographie du tourisme en 1981 (colloque d'Aix-en-Provence, 1981), [in:] Les Cahiers du Tourisme, C.H.E.T., 1982.

46. Les Monts des Sudètes et la demande internationale en sports d'hiver. Colloque polono-tchécoslovaque de Jelenia Góra, 1981, K.T.N., 1982.

47. Vars 1960-1980, 86 p., publié par la Mairie de Vars, 1982.

48. Croissance urbaine et extension spatiale dans les villes de la Région Provence-Alpes-Côte d'Azur, B.S.G.I., vol. XI, 1982.

49. Montagne, tourisme et aménagement en Bulgarie; communication à la Table Ronde du Groupe de travail «Pays de l'Est», Montpellier, 1982.

50. L'impact des activités humaines (stations de sports d'hiver) sur le milieu montagnard, B.A.G.F., mai 1982.

51. Les stations de sports d'hiver françaises et le milieu physique (Colloque franco-polonais de Kozubnik), Geo. Polo.,1983.

52. Puy Saint Vincent, une petite commune des Hautes-Alpes en face des sports d'hiver; rapport pour la Mairie, 1982 et 1984, en coll. avec L. Coulet.

53. Le tourisme dans les petites îles; rapport de base, colloque de la Commission du Tourisme de l'U.G.I., 1982 (Iles Lipari), [in:] «Le tourisme dans les petites îles», un vol., Messine, 1984.

54. Les industries nées du ski en France; ibidem (1982 et 1984).

55. Eaux de vie et alcools en Europe Orientale, première approche; communication au colloque sur l'histoire et la géographie des eaux-de-vie et vins, Cognac, 1982; publié par le C.E.R.V.I.N., Université de Bordeaux III, 1983.

56. Agriculture et tourisme dans un type de zone marginale: la montagne française. Communication au Congrès National des Géographes Italiens, Catane, 1983, [in:] Actes du Congrès.

57. L'impact des stations de ski sur le milieu naturel: bilan des recherches françaises; publications de la II $^{\text {ème }}$ Conférence Internationale du Tourisme, Banska Bystrica, 1983, en allemand.

58. La géographie des loisirs urbains et périurbains, Rapport général au Colloque de la Commission Française de géographie du tourisme, Angers, Nor., 1983.

59. La géographie régionale en France (avec coll.).

60. Equilibres et déséquilibres montagnards (avec P. Gabert). Deux études publiées par le Comité National Français de Géographie pour le Congrès de l'Union Géographique Internationale de Paris, 1984, [in:] «La géographie française», ouvrage collectif, Paris, 1984.

61. Vue d'ensemble sur le tourisme alpin, [in:] «Les Alpes», ouvrage collectif publié par le Comité National Français de Géographie pour le Congrès de l'U.G.I., Paris, 1984. 
62. La géographie du tourisme et des loisirs en France: définitions, conceptions et méthodes, principaux domaines d'étude, en coll. avec D. Pearce, in no spécial de Geojournal, 1984, préparé par la Commission de géographie du tourisme de l'U.G.I. (Président B. Barbier) pour le Congrès de Paris (1984) et consacré aux différentes conceptions de la géographie du tourisme (en anglais).

63. Les régions touristiques françaises, R.G.L., 1984, 1.

64. Le tourisme des grandes villes, présentation et conclusions. Colloque de la Commission tourisme U.G.I., Berlin, 1985; actes publiés par la Freie Universitat de Berlin Ouest en 1986.

65. Rapports villes-campagnes et réseau urbain, le point actuel; colloque franco-polonais de Sénanque, 1986, publié dans les «Cahiers de Fontenay», E.N.S. de Fontenay, 1987.

66. Le tourisme récepteur des grandes villes et métropoles économiques françaises, B.S.G.M., 1986.

67. La notion de réseau urbain dans la géographie régionale française, in ouvrage pour le Jubilé du Professeur L. Straszewicz, Łódź, 1986.

68. Réflexions sur le tourisme méditerranéen, [in:] Mélanges jubilaires offerts au Professeur J. Beaujeu-Garnier, (Régions, villes et aménagement), publiés par la Société de Géographie (de Paris) et le C.R.E. P.I.F., 1987.

69. In «Problemy Turystyki», revue polonaise de l'Instytut Turystyki (Varsovie), B. Barbier a été l'éditeur invité pour un no spécial consacré à «Formes nouvelles ou méconnues du tourisme», 1987, $2^{\text {ème }}$ trimestre et y a publié trois articles :

Le tourisme aujourd'hui ; permanences et changements.

Le tourisme des grandes villes.

Le tourisme des Japonais chez eux.

70. Les Calanques et leur massif, B.S.G.M., 1988.

71. Le rôle des acteurs locaux et régionaux dans le développement local et régional en Pologne; communication au colloque du Groupe de travail des Pays de l'Est, 1987, [in:] "Régions et pouvoirs régionaux en Europe de l'Est et en U.R.S.S.», éd. Masson, Paris, 1989.

72. Le tourisme rural montagnard. Le cas des Hautes-Alpes, Méd., 1989, 4.

73. A propos d'un livre récent, les noms de lieux en montagne, B.S.G.M., 1989.

74. Le tourisme international, une approche géographique; communication au colloque de la Commission du tourisme de l'U.G.I., Christchurch, 1988; publié in Geojournal, 1990, en anglais.

75. Attraits et milieux touristiques, in Mélanges offerts au Professeur J. Kostrowicki, Geo. Polo., 57, 1990.

76. Le tourisme rural en France; colloque franco-polonais Aix-Łódź, 1988, publié in Acta Universitatis Lodziensis, 1990.

77. Villes et régions dans la C.E.E. à la fin du XXème siècle, communication au colloque franco-polonais de Szymbark, 1989, publiée dans Geo. Polo., 1990.

78. Les stations de ski alpin: un cas de géographie urbaine, 1990; [in:] Actes du Congrès National des Socié-tés Savantes, 1991, Paris (Ministère de l’Education Nationale).

79. L'Europe Centrale, une définition géographique; communication au Colloque: "L'Europe Centrale. Réalités, mythes, enjeux, XVIII-XXèmes siècles"; publièe dans un ouvrage sous ce titre et édité en 1991 par l'Université de Varsovie (Institut Français), en français, et parue in Przegląd Geograficzny, en 1992 (en polonais).

80. La frontière orientale de la Pologne, in Mélanges offerts au Professeur L.Cassassas (Treballs de la Societat Catalana de Geografia, no spécial, déc 1992), Barcelone.

81. Les J. O. d'hiver à Albertville, B.S.G.M., 1992.

82. Francja, Belgia, Holandia, Luksemburg, Niemcy, [in:] Geografia świata, un vol. publié sous la direction de M. Rościszewski et édité par la Wydawnictwa Szkolne i Pedagogiczne, Warszawa, 1992.

83. Le catholicisme en Pologne aujourd'hui, Ac.de M., 1992.

84. Problèmes polonais d'aujourd'hui, Ac.de M., 1993.

85. Stations de ski ou grands domaines skiables?, in Mélanges offerts au Professeur P. Gabert ("Géomorphologie et aménagement de la montagne»), éd. par le Centre de géomorphologie du C.N.R.S. de Caen, 1993.

86. Le tourisme dans le Moyen Atlas marocain, colloque de Missour (Maroc), 1994.

87. Histoire de la Provence, tome IV, quatrième partie: "A l'aube du troisième millénaire", p. 263 à 340.

88. Patrimoine, patrimoine mondial (U.N.E.S.C.O.) et tourisme, Turyzm, t. 5, z. 2, Łódź, 1995. 
89. L'évolution socio-économique de l'Ile Saint-Louis de 1962 à 1995, [in:] "L'Ile Saint-Louis", publication du Service de la Direction Artistique de la Ville de Paris, un vol., 1997.

90. La Pologne, Coll. "Que sais-je?», un vol., 1998.

91. Problèmes géopolitiques de la Pologne, [in:] Geopolitical studies (publication de l'I.G.P.Z - P.A.N.), 1999 , en polonais.

92. L'Arménie d'aujourd'hui, B.S.G.M., 2000.

\section{OBJAŚNIENIA SKRÓTÓW/ABREVIATIONS}

Ac.de M. - Actes de l'Académie de Marseille.

Ann. de G. - Annales de Géographie, A. Colin, Paris.

B.A.G.F. - Bulletin de l'Association de Géographes Français.

B.S.E.H.A. - Bulletin de la Société d'Etudes des Hautes-Alpes (Gap).

B.S.G.I. - Boletino della Societa Geographica Italiana.

B.S.G.M. - Bulletin de la Société de Géographie de Marseille.

B.S.G.S.S. - Bulletin de la section de géographie (Congrès des Sociétés Savantes).

C.H.E.T. - Publication du Centre des Hautes Etudes du tourisme (Université d'Aix-en- Provence).

Doc.Fr. - La Documentation Française, Paris.

Et.V. - Etudes Vauclusiennes (Université d'Avignon).

Géo.Polo. - Geographia Polonica, revue de géographie de l'Académie Polonaise des Sciences.

H.T.du.N. - Hommes et Terres du Nord, revue de géographie de l'Université de Lille.

I.G. - Information Géographique, Paris.

K.T.N. - Bulletin de la Karkonoskie Towarzystwo Naukowe (Jelenia Góra).

Méd. - Méditerranée (Institut de Géographie, Université de Provence).

Nor. - Norois, revue de géographie (Universités de l'Ouest de la France).

R.C.C.M. - Revue de la Chambre de Commerce de Marseille.

R.G.A. - Revue de Géographie Alpine (Université de Grenoble III ).

R.G.L. - Revue de Géographie de Lyon (Université de Lyon II ).

R.G.G.R. - Revue de Géographie et de Géologie de Roumanie.

TUR. - - Turyzm. Revue de l'Institut de Géographie des Villes et du Tourisme de l'Université de Łódź.

W.G.S. - Wiener Geographische Schriften (Université de Vienne). 